\title{
Postbariatric EArly discharge Controlled by Healthdot (PEACH) trial: study protocol for a preference-based randomized trial
}

\author{
Jai Scheerhoorn ${ }^{1 *}$ (D) Lisa van Ede ${ }^{2}$, Misha D. P. Luyer ${ }^{1}$, Marc P. Buise ${ }^{2}$, R. Arthur Bouwman ${ }^{2,3}$ and \\ Simon W. Nienhuijs ${ }^{1}$
}

\begin{abstract}
Introduction: Performing bariatric surgery in a daycare setting has a potential reduction in hospital costs and increase in patients' satisfaction. Although the feasibility and safety of such care pathway has already been proven, its implementation is hampered by concerns about timely detection of short-term complications. This study is designed to evaluate a combined outcome measurement in outpatient bariatric surgery supplemented by a novel wireless remote monitoring system versus current standard of care.

Methods and analysis: A total of 200 patients with multidisciplinary team approval for primary bariatric surgery will be assigned based on their preference to one of two postoperative trajectories: (1) standard of in-hospital care with discharge on the first postoperative day or (2) same day discharge with ongoing telemonitoring up to 7 days after surgery. The device (Healthdot R Philips) transfers heart rate, respiration rate, activity, and body posture of the patient continuously by LoRaWan network to our hospital's dashboard (Philips Guardian). The primary outcome is a composite outcome measure within 30 days postoperative based on mortality, mild and severe complications, readmission, and prolonged length-of-stay. Secondary outcomes include patients' satisfaction and data handling dimensions.
\end{abstract}

Trial registration: ClinicalTrials.gov NCT04754893, Registered on 12 February 2021.

Keywords: Bariatric surgery, Telemonitoring, Outpatient surgery, Home monitoring, Patient preference randomized trial, Study protocol

\section{Background}

Bariatric surgery has proven its worth for the treatment of obesity and is considered to be the most effective long-term treatment option [1-3]. Implementation of

\footnotetext{
* Correspondence: jai.scheerhoorn@catharinaziekenhuis.nl

Name and contact information for the trial sponsor: Liesbeth Vonk, Study Manager - Design Research, liesbeth.vonk@philips.com.

Role of study sponsor: Philips Electronic Nederland BV has played a role in the design of this study. They will not play any role in the management, analysis, and interpretation of data; writing of the report; or the decision to submit the report for publication. They will have no ultimate authority over any of these activities.

'Department of Surgery, Catharina Hospital, Eindhoven, The Netherlands Full list of author information is available at the end of the article
}

Enhanced Recovery After Bariatric Surgery (ERABS) reduced the length of hospital stay (LOS) without significant influence on overall morbidity $[4,5]$. Furthermore, it reduced risk of hospital-acquired infections, improved accessibility to bariatric surgery, improved patient and health professional satisfaction, and increased costeffectiveness [6, 7]. Current standard of care in The Netherlands is to discharge patients 1 day postoperatively [8].

Literature has proven the safety of performing outpatient bariatric surgery such as laparoscopic gastric sleeve gastrectomy (SG) and Roux-en-Y gastric bypass

(c) The Author(s). 2022 Open Access This article is licensed under a Creative Commons Attribution 4.0 International License, which permits use, sharing, adaptation, distribution and reproduction in any medium or format, as long as you give appropriate credit to the original author(s) and the source, provide a link to the Creative Commons licence, and indicate if changes were made. The images or other third party material in this article are included in the article's Creative Commons licence, unless indicated otherwise in a credit line to the material. If material is not included in the article's Creative Commons licence and your intended use is not permitted by statutory regulation or exceeds the permitted use, you will need to obtain permission directly from the copyright holder. To view a copy of this licence, visit http://creativecommons.org/licenses/by/4.0/ The Creative Commons Public Domain Dedication waiver (http://creativecommons.org/publicdomain/zero/1.0/) applies to the data made available in this article, unless otherwise stated in a credit line to the data. 
(RYGB) [4, 9-14]. Despite being proven to be safe, the implementation of outpatient bariatric surgery is not yet widespread. Underlying reason relates to the concern that short-term complications such as bleeding may not be timely be detected $[15,16]$. Most often these complications first express themselves by a change in vital parameters such as tachycardia. A wireless remote monitoring system would allow monitoring of vital signs in patients that have been discharged shortly following surgery and facilitate detection of early postoperative complications in a home environment. Moreover, telemonitoring could also be of added value to detect lateterm complications which occur in a home situation.

Even though many wireless monitoring devices are available, most are still in clinical validation and feasibility testing phases [17], and bear the disadvantage that vital signs data have to be uploaded via a datahub. Experience from previous studies learnt that home monitoring was hampered in the home situation by difficulties with regard to connectivity issues and ease of use leading to incomplete data acquisition. New clinically tested and validated devices have become available, such as Healthdot (Philips Electronic Nederland BV), that continuously collect vital signs and supports automated data transmission towards a vital signs dashboard in the hospital and as such increasing ease of use and patient comfort [18]. Using this device it seems possible to discharge patients on the day of surgery without compromising the early detection of short-term complications.

The primary objective of this patient preferencebased randomized trial is to that implementation of ambulatory bariatric surgery combined with remote monitoring up to 7 days after surgery using a wireless unobtrusive continuous monitoring device with automated data transmission, is not inferior to the current standard postoperative care, while maintaining patient comfort and satisfaction.

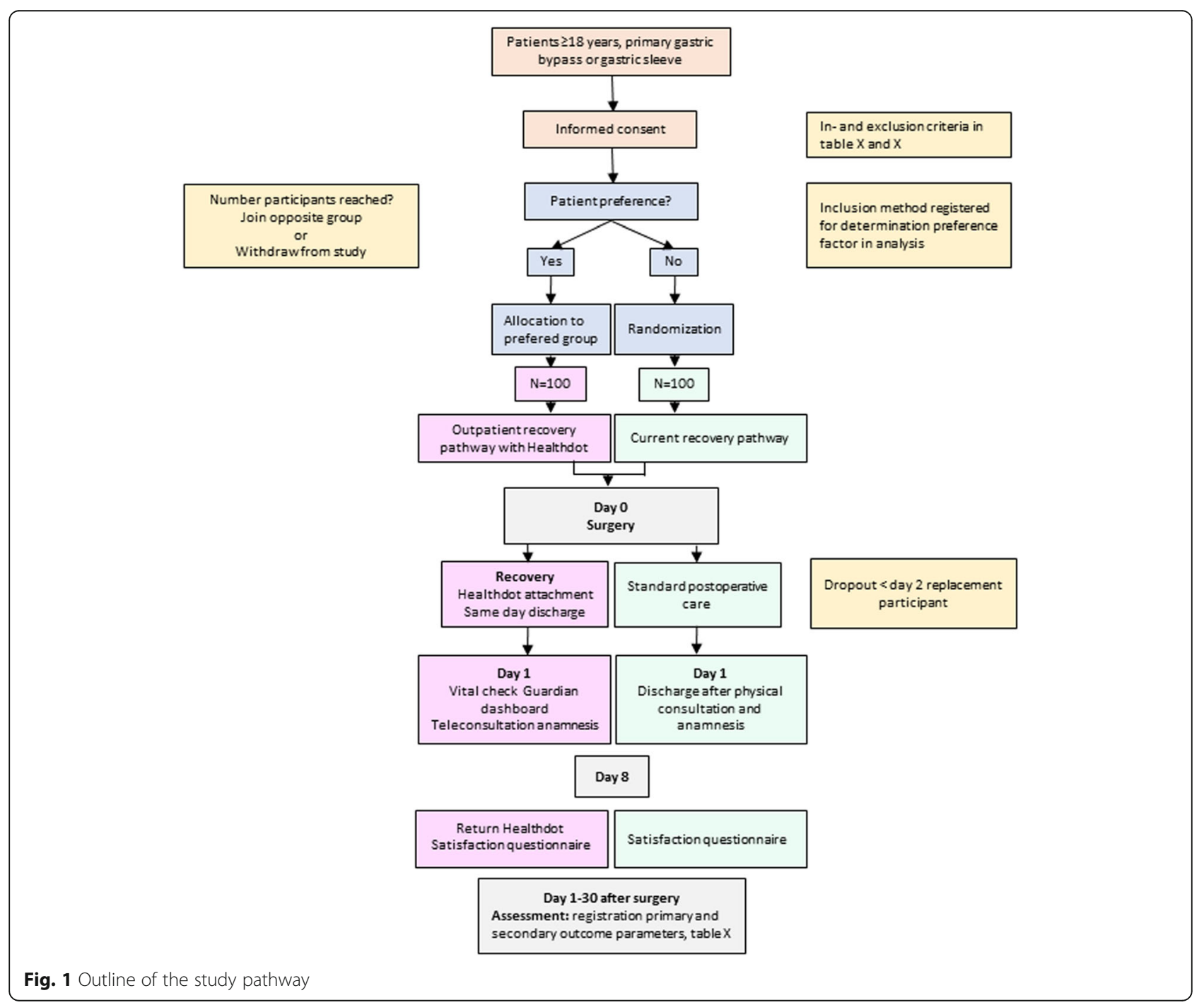




\section{Methods and analysis}

\section{Study design}

This single-center preference-based randomized trial in a tertiary hospital in the Netherlands is designed to compare the outcomes of two different recovery pathways after standard care bariatric surgery. Figure 1 shows an outline of the study pathway.

Primary outcome is a combined measurement of mortality, severe postoperative complications (Clavien-Dindo IIIb or higher), mild complications (Clavien-Dindo II and IIIa), hospital readmission, or prolonged length of stay, all within 30 days after surgery. Secondary outcomes include patients' satisfaction, data handling dimensions, post-operative analgesia, and costeffectiveness.

Randomized controlled trials (RCT) are suggested to provide the most reliable evidence for treatment efficacy [19]; however, treatment preferences can decrease the generalizability of the results and reduce its external validity. Nevertheless, recent literature has shown the added value of including patient preference in medical research [20-22]. Throughout the years, various alternative designs have been suggested to diminish the influence of patients' preference on validity. One of these approaches is the partially randomized patient preference trial (RPPT). Recently, a systematic review has shown patients preference did not influence the primary outcome despite of a substantial proportion of a specific patient group refusing randomization [19]. Therefore, compared to RCTs, a RPPT can increase external validity without compromising the internal validity. In current daily clinical practice, patient preference has a significant impact on decisions made by clinicians regarding their treatment or treatment pathway. Hence we believe that implementation of this new recovery pathway comes to its best when mimicking daily practice as much as possible. Therefore, in our opinion, an RPPT is best suitable to investigate our endpoints.

\section{Study population}

The patient is eligible to participate in the study if they are planned for a primary laparoscopic gastric bypass or gastric sleeve. Inclusion and exclusion criteria are mentioned in Tables 1 and 2, respectively. Any patient referred to our center is screened by a multidisciplinary

Table 1 Inclusion criteria

Willing and able to sign informed consent form

Able to understand instructions

An adult person must be present at the same location as the patient during the first night following surgery who is able to mobilize help or seek medical care if necessary

In possession of a telephone on which patient can be reached for the duration of participation (days 1-8)
Table 2 Exclusion criteria

Patients of psychiatric wards, inmates or prisons, or other state institutions

Investigator or any other team member involved directly or indirectly in the conduct of the clinical study

Any skin condition, for example, prior rash, discoloration, scars, or open wounds at the area where the Healthdot needs to be placed

Known allergy for the tissue adhesive used in the Healthdot (white band-aid)

Use of topical that is known to influence the skin at the test area (such as medical and non-medical creams or lotions)

Patient with active implantables such as Implantable Cardioverter Defibrillator (ICD) and pacemaker

Left lower rib (place where Healthdot will be applied) is involved in the area of surgery, area of disinfection, or area where bandages are needed.

Expected participation less than 8 days

team for eligibility for bariatric surgery. They are asked to fill out screening questionnaires and receive presentation and interviews by a dietician, obesity nurse, psychologist, and physiotherapist and receive extensive blood tests. The combined findings of this screening are discussed in a multidisciplinary session in which the surgeon is also involved. When approved, patients visit the bariatric surgeon to determine which bariatric procedure is deemed most suitable. After informed consent has been given, it will be determined whether the patient prefers the current standard of care or the outpatient recovery pathway. Patients who choose the outpatient pathway have to make sure there is someone with them the night after the surgery who is able to contact the hospital when questions arise and need to be able to mobilize help in case of a medical emergency. The factor of preference will be included in the analysis. If the preferred group is full, the patient can choose either to participate in the other group or withdraw from the study. The patients without preference are randomized in a 1:1 ratio. Simple randomization is used, meaning every individual has an equal chance to be enrolled in one of the two groups. Allocation is into one of two groups: (1) outpatient group $(n=100)$ or (2) current standard of care group $(n=100)$. Patients will be randomly assigned through a computer-generated randomization program. This program will take into account group size to end up with an equal number. Allocation concealment for patients without preference will be ensured, as the allocation of patients is generated after the patient signs the informed consent form and thereby included in the study

Patients who change their minds (change preference) before surgery can switch to the other study group. If a patient changes his/her preference between surgery and discharge this will be noted as a failure to implement the 
new protocol. In case same day discharge is not deemed feasible for surgical and/or medical reasons at the discretion of the attending surgeon or anesthesiologist, the patient will be noted as failure as well. Any change in patient preference/study group will be registered. Any other situation will be considered as a dropout (Table 3).

\section{Outcome measures}

The primary outcome of the study is the absence of the following within 30 days after primary surgery.

1. Mortality

2. Severe postoperative complications (Clavien-Dindo IIIb or higher)

3. Readmission (at least one night in hospital)

4. Mild complications (Clavien-Dindo II and IIIa)

5. Prolonged length of stay ( 3 days or more in hospital)

Secondary outcome measures are shown in Table 4 .

\section{Sample size calculation}

Sample size calculation was based on a previous study in the Netherlands in which the primary outcome measure was used [23]. In this study, the average outcome across bariatric surgery patients in the Netherlands was $88.7 \%$. In current practice in the Catharina Hospital, Eindhoven, 95\% of all bariatric surgery patients meet this combined outcome measure. This helps to derive a non-inferiority margin of around 7\%. A $p$ value of 0.05 will be considered statistically significant.

Sample size calculations were performed using PS: Power and Sample Size Calculation software (v3.1.2, Vanderbilt University). With a power of 0.80 , an $\alpha$ of 0.05 , a non-inferiority margin of 0.07 , and an expected proportion of 0.96 , this resulted in a sample size of 97 patients per group using an uncorrected chi-squared statistic.

The expected drop-out rate is 10\% (20 patients in this sample). Patients dropping out before study start or during the first 2 days of the study will be replaced until the final 200 patients are included and have participated more than the 2 days defined for replacement.

The interim analysis will take place when data from 50 patients has been collected. The data of this study will not be monitored during the study. The study will be monitored. Monitor visits will occur after; 15 patients

Table 3 Dropout criteria

Patients who are unwilling to further participate in the study

Patients who did not undergo surgery (for example new onset of disease which makes the patient unsuitable for surgery)

Concomitant new onset of a disease influencing the outcome
Table 4 Secondary outcome measures

Patient satisfaction $>6$ points (on a scale of 1-10)

Percentage of patients recruited for the outpatient recovery after standard bariatric surgery group

Percentage of patients with full adherence to protocol and randomization

Percentage of missing data

Percentage of false positive of the positive notifications from the Healthdot system

Total number of false positive of the positive notifications from the Healthdot system

Total number of false negative notifications from the Healthdot system

The outcome of patients who choose HD versus patients who were randomized to $\mathrm{HD}$ on a combined outcome measure as defined for the primary endpoint.

To evaluate the outcome of patients who choose HD versus patients who were randomized to HD on a combined outcome measure as defined for the primary endpoint.

Number of adverse events in both groups

Amount of pain medication taken on the day of surgery in both groups Percentage of clinical decisions made based on the Healthdot system, that changed when information from a telephone consultation was also included.

Patient and health professional satisfaction (on a scale from 1 to 10) for both the outpatient recovery after standard bariatric surgery group as the current recovery path group

The costs involved with outpatient recovery after standard bariatric surgery supported by Healthdot and the cost involved with the current recovery path, in the period up to 7 days after surgery.

have been included, 50 patients have completed followup, and once all patients have completed follow-up.

\section{Recruitment}

Participant recruitment will occur in our outpatient clinic. Every patient approved for bariatric surgery will be approached to participate by one of the study members. With around 900 bariatric procedures a year in the Catharina Hospital, the supply of eligible patients is large enough. Given the design of our study in which patients can decide in which group to participate, we believe that the participation rate will be high.

\section{The Healthdot system}

The Healthdot system (Philips Electronic Nederland BV) consists of a wearable data logger that transmits data to be visualized real-time on our hospital dashboard after pairing the Healthdot to the patient. The data logger device consists of an adhesive layer, electronics, and a battery (Fig. 2). The general operating principle is based on the continuous collection and periodic transmission of heart rate, respiratory rate, activity, and posture by means of processing of accelerometer signals. Every 5 min, the mean of the data is transmitted from the Healthdot device to a Cloud Server (Health Suite Digital 


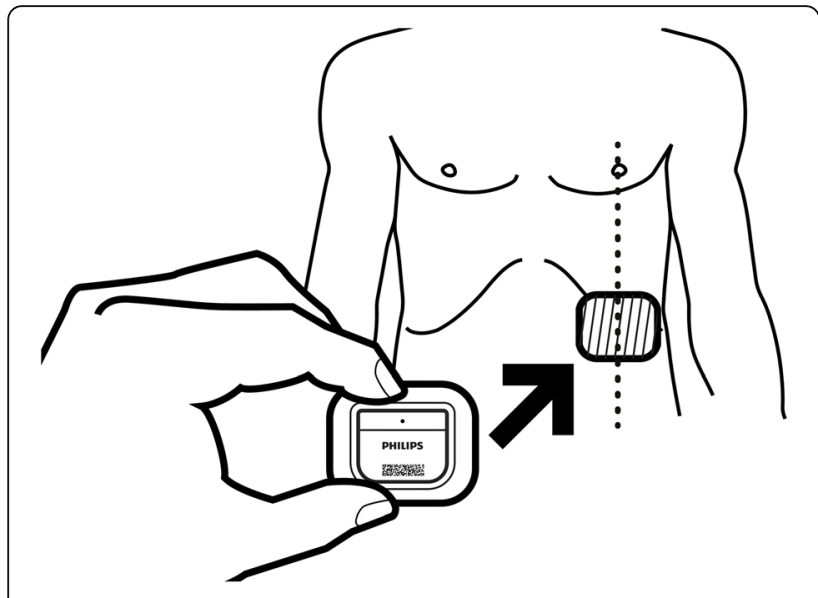

Fig. 2 Schematic view of the Healthdot. Reprinted from Philips Electronic Nederland BV under a CC BY license, with permission from Philips Electronic Nederland BV, original copyright 2020

Platform, HSDP, Philips electronics Nederland bv) using the KPN LoRaWan network, as a part of the Philips Guardian System.

\section{The Guardian Dashboard}

Data of the parameters (heart rate, respiration rate, activity, and body posture) will be available to view in the in hospital Guardian Dashboard (Philips Intellivue Guardian: release E, Enterprise release 13.1). Once logged into the system, the vitals are presented as trend figures. Events with a heart rate or respiration rate above a pre-defined threshold will be flagged and a warning score will be calculated. There will be no alarm notifications when not logged into the system.

Literature has shown that the heart rate of patients after bariatric surgery complicated by either anastomotic leakage or postoperative bleeding is $>120$ beats per minute (bpm) in respectively $87.5 \%$ vs $35 \%$ and $>100-120$ bpm in $12,5 \%$ and $60 \%$ of the patients [24-26]. Based on literature and own experience of the medical team, the threshold was set at a heart rate above 110 beats per minute (bpm) and a respiration rate above 20 with a reassurance period of $15 \mathrm{~min}$.

\section{Intervention}

\section{Start of the study}

Patients who have chosen to undergo outpatient surgery will be scheduled for surgery on the earliest available time-slots in the morning. The Healthdot will be applied mid-clavicular on the lowest left rib of the chest as the patient is in the recovery room. The Healthdot can be removed by the patient themselves in case of emergency with a remover tissue which will be handed out at discharge. The postoperative care will be arranged in such a way that same day discharge is possible for this group of patients. Patients who have chosen the regular treatment will receive care in accordance with the current protocol.

\section{Discharge from hospital}

At the end of the day, patients in the outpatient surgery group will be discharged from the hospital on the discretion of a nurse under supervision of a physician, wearing the Healthdot. Patients will only be discharged if they meet the following criteria: pain should be adequately controlled with oral medication, there should be no nausea or vomiting, the patient is mobilizing and the patient is willing to be discharged. Furthermore, clinical findings should show no fever, a heart rate $\leq 110 \mathrm{bpm}$, and an adequate oral intake. Laboratory findings should show a no hemoglobin decrease of more than $2 \mathrm{mmol} / \mathrm{l}$ when compared to the preoperative value. The physician will be able to view the parameters on the Guardian dashboard when measured by Healthdot at all times when logged into the system, including the flag marks and warning scores. A teleconsultation will be scheduled for the following morning (day 2). Before the teleconsultation, the vital signs in the dashboard will be interpreted by the physician who will then assess whether additional action is indicated. The decision based on Healthdot data is noted. After the teleconsultation, it is noted whether additional or different decisions are made about the treatment based on the additional information from the teleconsultation.

On days $3-8$, the physician or a nurse will check the Guardian dashboard daily. The physician may decide to have a telephone consultation, e.g., when the Healthdot dashboard flags an event. If the physician determines that additional action is needed (e.g., based on the information on the dashboard, or when the patient calls), in the absence of an indication from the Healthdot, such an event will be viewed as a false negative. It will be retrospectively assessed, based on the patient medical record, if false negative events have occurred. For all events flagged in the Healthdot dashboard, the physician will record whether it was a false positive notification or a true positive notification.

Patients in the regular treatment group will be released from the hospital at the discretion of the physician the day after surgery. For these patients, no appointments outside of the regular protocol are planned.

\section{Follow-up}

Patients who received the Healthdot will remove this themselves and are requested to send the device back to the hospital on day 8. At hospital discharge, the patient will be handed out a return envelope including the return instructions. The envelope also includes a patient/ healthcare satisfaction questionnaire they are requested 
to fill in and send along with the device. The presence or absence of readmission within 30 days will be observed from the patient record and collected in a anonymized database. Health care professionals will also be requested to fill in a short satisfaction questionnaire after the end of the study.

The schedule of this trial is shown in Fig. 3.

\section{Discussion}

In this study we aim to demonstrate bariatric surgery as outpatient surgery combined with remote monitoring is non-inferior to the current standard postoperative care, while patient comfort and satisfaction is maintained.

Healthcare has developed rapidly over the last decades. The implementation of ERAS protocols in bariatric surgery has led to improved outcomes and has shortened length of stay to the point where bariatric surgery is being performed as outpatient procedures [4, 5, 8]. Recent technological development has led to novel devices that can wirelessly monitor the vital parameters of a patient in the hospital as well as at home. Therefore, in theory, patients no longer need to stay admitted to be able to detect post-operative complications. This is the first patient preference-based randomized trial in which bariatric surgery will be performed as an outpatient procedure and the patients' vital parameters will be monitored at home using a novel device. There are some studies that use the surgical patient as a research population. However, these are usually studies which take place in-hospital or in which the patient has been in the hospital for several days between surgery and discharge [27-30]. Therefore,

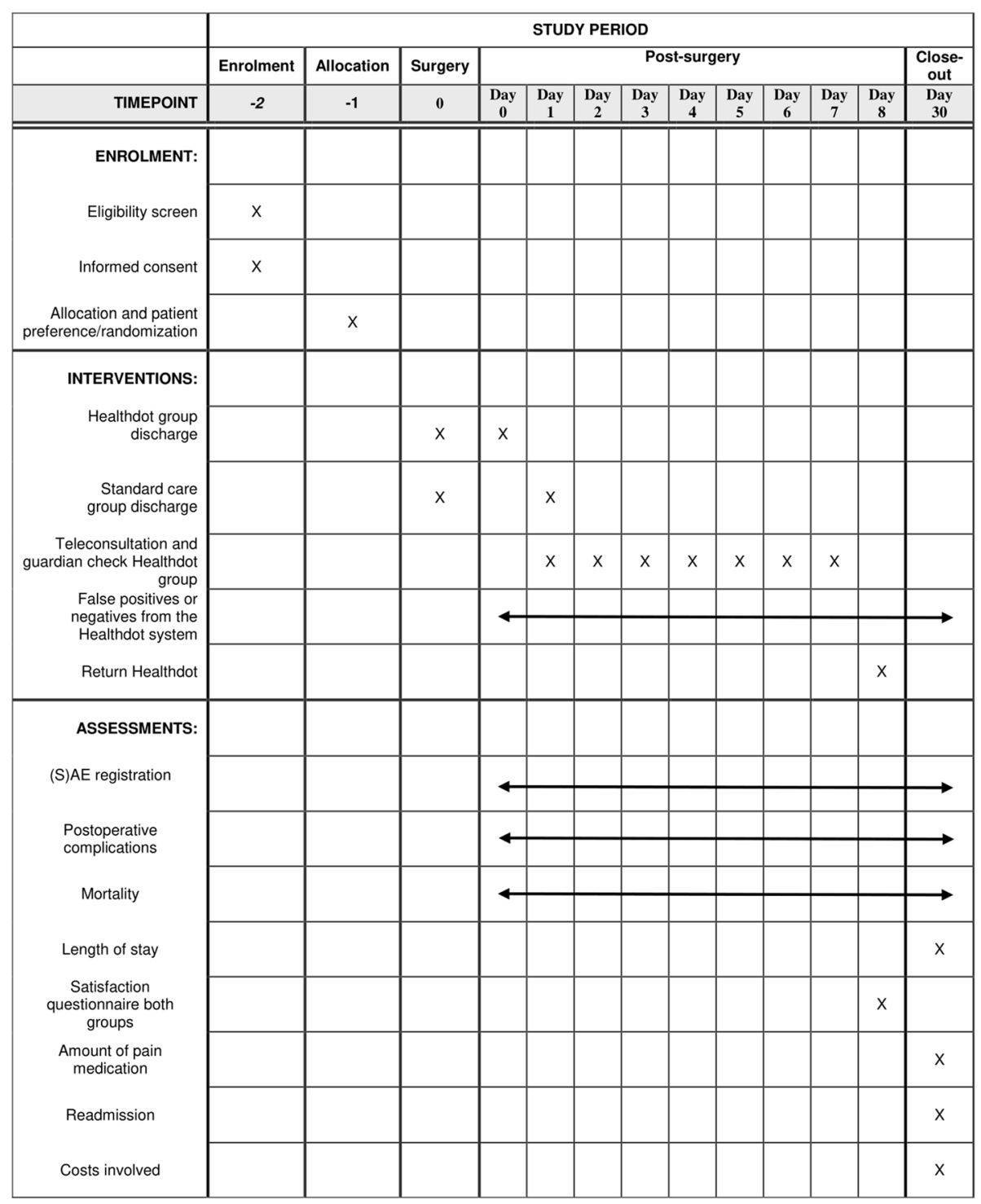

Fig. 3 Schedule of this trial 
these studies cannot be directly compared with this study.

Some limitations need to be addressed as well. Being a patient preference-based randomized trail instead of a randomized control trial (RCT), the patient being able to choose his or her treatment can lead to bias. For example, it is possible that young patients are more inclined to participate in the outpatient treatment group then older patients. This can impact outcomes and will need to be taken into consideration during statistical analysis. Although, as mentioned previously, recent studies have shown that compared to RCTs, a RPPT can increase external validity without compromising the internal validity [19].

Using a relatively new medical device (Healthdot) in this study can also influence results. The impact of falsepositives or negatives from the Healthdot system on the outcomes is unknown. The notification thresholds used in this study are based on a combination of literature and own experience but are not yet defined on analysis. This study will provide insight in data whereby a protocol like this can be adjusted and specified. Furthermore, the study requires a new treatment pathway to be implemented in daily clinical practice which could take time and effort from nurses and other health workers.

This study will give insights into the safety of outpatient bariatric surgery when combined with telemonitoring in a large single-center clinical trial. It will also shed light on patient satisfaction when performing bariatric surgery on an outpatient basis compared to the current standard and, furthermore, can answer the question if costs can be saved by implementing this new pathway.

\section{Acknowledgements}

None

\section{Trial status}

Protocol version 1.0, 16-02-2021

Estimated Study Start Date: February 22, 2021

Estimated Study Completion Date: July 2021

\section{Ancillary and post-trial care}

Upon signing informed consent participants are given the option if their data can be used for future research.

\section{Authors' contributions}

All named authors adhere to the authorship guidelines of Trials. All authors have agreed to the publication. JS, RB, and SN designed the study. JS, LE, RB, and SN drafted the manuscript. ML and MB edited the manuscript. The authors read and approved the final manuscript.

\section{Funding}

Philips Electronic Nederland BV (trial sponsor) will supply the medical device needed (Healthdot) for the execution of this study.

\section{Availability of data and materials}

All investigators will have access to the datasets. The project datasets will be hosted on the hospital's secure network and all datasets will be password protected. Anonymized data will be available for Philips Electronics Nederland B.V. for development purposes.

\section{Declarations}

\section{Protocol amendments}

In the case of a necessary protocol amendment, the amendment will be submitted to the ethics committee.

\section{Ethics approval and consent to participate}

Ethics approval has been obtained from the Medical Ethical Committee of the Maxima Medical Center (Reference number W20.095) who have reviewed and approved the study procedures, ethics, subject information and consent, and subject safety. The results of the trial will be submitted for publication in a peer-reviewed journal.

\section{Consent for publication}

Not applicable

\section{Competing interests}

$\mathrm{RB}$ acts as clinical consultant for Philips Research in Eindhoven, The Netherlands. This does not alter our adherence to BMC policies on sharing data and materials.

\section{Author details}

${ }^{1}$ Department of Surgery, Catharina Hospital, Eindhoven, The Netherlands. ${ }^{2}$ Department of Anesthesiology, Catharina Hospital, Eindhoven, The Netherlands. ${ }^{3}$ Department of Electrical Engineering, Eindhoven University of Technology, Eindhoven, The Netherlands.

Received: 19 April 2021 Accepted: 4 January 2022

Published online: 21 January 2022

\section{References}

1. Colquitt JL, Picot J, Loveman E, et al. Surgery for obesity. Cochrane database Syst. Rev. [Internet]. 2009;15(2):CD003641.

2. Picot J, Jones J, Colquitt $J \mathrm{~L}$, et al. The clinical effectiveness and costeffectiveness of bariatric (weight loss) surgery for obesity: a systematic review and economic evaluation. Health Technol. Assess. 2009;13:1-190 215-357, iii-iv.

3. Buchwald H, Estok R, Fahrbach K, et al. Weight and type 2 diabetes after bariatric surgery: systematic review and meta-analysis. Am. J. Med. Elsevier Inc. 2009:122:248-256.e5.

4. Leepalao MC, Arredondo D, Speights F, Duncan TD. Same-day discharge on laparoscopic Roux-en-Y gastric bypass patients: an outcomes review. Surg Endosc. 2019). https://doi.org/10.1007/s00464-019-07139-5;34(8):3614-7.

5. Lam J, Suzuki T. An ERAS protocol for bariatric surgery: is it safe to discharge on postoperative day 1? Surgical Endoscopy. 2019;33(2):580-6. https://doi. org/10.1007/s00464-018-6368-9.

6. Ahmed O, Rogers A, Robb WB, et al. Meta-Analysis of Enhanced Recovery Protocols in Bariatric Surgery. Journal of Gastrointestinal Surgery. 2018;22(6): 964-72. https://doi.org/10.1007/s11605-018-3709-x.

7. Khorgami Z, Petrosky JA, Andalib A, Aminian A, Schauer PR, Brethauer SA. Fast track bariatric surgery: safety of discharge on the first postoperative day after bariatric surgery. Surg Obes Relat Dis. 2017;13(2):273-80. https://doi. org/10.1016/j.soard.2016.01.034

8. Poelemeijer YQM, Liem RSL, Våge V, Mala T, Sundbom M, Ottosson J, et al. Perioperative Outcomes of Primary Bariatric Surgery in North-Western Europe: a Pooled Multinational Registry Analysis. OBES SURG. 2018). https:// doi.org/10.1007/s11695-018-3408-4;28(12):3916-22.

9. Surve A, Cottam D, Zaveri H, Horsley B, et al. Does the future of laparoscopic sleeve gastrectomy lie in the outpatient surgery center? A retrospective study of the safety of 3162 outpatient sleeve gastrectomies. Surg Obes Relat Dis. 2018 Oct;14(10):1442-7. https://doi.org/10.1016/j.soa rd.2018.05.027.

10. Billing PS, Crouthamel MR, Oling S, Landerholm RW. Outpatient laparoscopic sleeve gastrectomy in a free-standing ambulatory surgery center: first 250 cases. Surg Obes Relat Dis. 2014;10(1):101-5. https://doi. org/10.1016/j.soard.2013.07.005.

11. Aftab H, Fagerland MW, Nordby T, et al. Gastric sleeve resection as day-case surgery: what affects the discharge time. Surg Obes Relat Dis. 2019;15(12): 2018-24. 
12. Inaba CS, Koh CY, Sujatha-Bhaskar S, Pejcinovska M, Nguyen NT. How safe is same-day discharge after laparoscopic sleeve gastrectomy? Surg Obes Relat Dis. 2018;14(10):1448-53. https://doi.org/10.1016/j.soard.2018.07.016.

13. Morton JM, Winegar D, Blackstone R, Wolfe B. Is ambulatory laparoscopic Roux-en-Y gastric bypass associated with higher adverse events? Ann Surg. 2014:259(2):286-92. https://doi.org/10.1097/SLA.0000000000000227.

14. Inaba CS, Koh CY, Sujatha-Bhaskar S, Zhang L, Nguyen NT. Same-day discharge after laparoscopic RouX-en-Y gastric bypass: an analysis of the Metabolic and Bariatric Surgery Accreditation and Quality Improvement Program database. J Am Coll Surg. 2018;226(5):868-73. https://doi.org/10.1 016/j.jamcollsurg.2018.01.049.

15. Goitein D, Raziel A, Szold A, et al. Assessment of perioperative complications following primary bariatric surgery according to the Clavien-Dindo classification: comparison of sleeve gastrectomy and Roux-Y gastric bypass. Surg Endosc. 2016;30:273-8.

16. Zellmer JD, Mathiason MA, Kallies KJ, Kothari SN. Is laparoscopic sleeve gastrectomy a lower risk bariatric procedure compared with laparoscopic Roux-en-Y gastric bypass? A meta-analysis. Am J Surg. 2014;208(6):903-10. https://doi.org/10.1016/j.amjsurg.2014.08.002.

17. Leenen JPL, Leerentveld C, van Dijk JD, van Westreenen HL, Schoonhoven L, Patijn GA. Current Evidence for Continuous Vital Signs Monitoring by Wearable Wireless Devices in Hospitalized Adults: Systematic Review. J Med Internet Res. 2020;22(6):e18636. https://doi.org/10.2196/18636.

18. Jacobs F, Scheerhoorn J, Mestrom E, et al. Reliability of heart rate and respiration rate measurements with a wireless accelerometer in postbariatric recovery. Under revision (since December 2020). PLOSONE. 2021;16(4):e0247903.

19. Wasmann KA, Wijsman P, van Dieren S, Bemelman W, Buskens C. Partially randomised patient preference trials as an alternative design to randomised controlled trials: systematic review and meta-analyses. BMJ Open. 2019; 9(10):e031151. https://doi.org/10.1136/bmjopen-2019-031151.

20. van Overbeeke E, Janssens R, Whichello C, Schölin Bywall K, Sharpe J, Nikolenko N, et al. Design, Conduct, and Use of Patient Preference Studies in the Medical Product Life Cycle: A Multi-Method Study. Front Pharmacol. 2019;10:1395. https://doi.org/10.3389/fphar.2019.01395 PMID: 31849657; PMCID: PMC6902285.

21. Ho MP, Gonzalez JM, Lerner HP, Neuland CY, Whang JM, McMurry-Heath M, et al. Incorporating patient-preference evidence into regulatory decision making. Surg Endosc. 2015;29(10):2984-93. https://doi.org/10.1007/s00464014-4044-2 Epub 2015 Jan 1. PMID: 25552232.

22. Egbrink MO, IJzerman M. The value of quantitative patient preferences in regulatory benefit-risk assessment. J Mark Access Health Policy. 2014;2. https:// doi.org/10.3402/jmahp.v2.22761 PMID: 27226836; PMCID: PMC4865769.

23. Poelemeijer YQM, Marang-van de Mheen PJ, Wouters MWJM, Nienhuijs SW, Liem RSL. Textbook Outcome: an Ordered Composite Measure for Quality of Bariatric Surgery. Obes Surg. 2019;29(4):1287-94. https://doi.org/10.1007/ s11695-018-03642-1.

24. Bellorin O, Abdemur A, Sucandy I, Szomstein S, Rosenthal RJ. Understanding the significance, reasons and patterns of abnormal vital signs after gastric bypass for morbid obesity. Obes Surg. 2011;21(6):707-13. https://doi.org/1 0.1007/s11695-010-0221-0 PMID: 20582574.

25. Sucandy I, Martin G, Szomstein S, Rosenthal R. Reasons for tachycardia in the postoperative period of patients undergoing laparoscopic Roux-en-Y gastric bypass. Surg Obes Relat Dis. 2009;5(3):S36-7. https://doi.org/10.1016/ j.soard.2009.03.105

26. Hamilton E, Sims T, Hamilton T, et al. Clinical predictors of leak after laparoscopic Roux-en-Y gastric bypass for morbid obesity. Surg Endosc. 2003). https://doi.org/10.1007/s00464-002-8819-5;17(5):679-84.

27. Leenen JPL, Dijkman EM, van Dijk JD, et al. Feasibility of continuous monitoring of vital signs in surgical patients on a general ward: an observational cohort study. BMJ Open. 2021;11(2):e042735. https://doi.org/1 0.1136/bmjopen-2020-042735 PMID: 33597138; PMCID: PMC7893648.

28. Breteler MJM, KleinJan EJ, Dohmen DAJ, et al. Vital Signs Monitoring with Wearable Sensors in High-risk Surgical Patients: A Clinical Validation Study. Anesthesiology. 2020;132(3):424-39. https://doi.org/10.1097/ALN. 0000000000003029 PMID: 31743149.

29. Downey C, Ng S, Jayne D, Wong D. Reliability of a wearable wireless patch for continuous remote monitoring of vital signs in patients recovering from major surgery: a clinical validation study from the TRaCINg trial. BMJ Open. 2019;9(8):e031150. https://doi.org/10.1136/bmjopen-2019-031150 PMID: 31420399; PMCID: PMC6701670.
30. Breteler MJM, Numan L, Ruurda JP, et al. Wireless Remote Home Monitoring of Vital Signs in Patients Discharged Early After Esophagectomy: Observational Feasibility Study. JMIR Perioper Med. 2020;3(2):e21705. https:// doi.org/10.2196/21705 PMID: 33393923; PMCID: PMC7728408.

\section{Publisher's Note}

Springer Nature remains neutral with regard to jurisdictional claims in published maps and institutional affiliations.
Ready to submit your research? Choose BMC and benefit from:

- fast, convenient online submission

- thorough peer review by experienced researchers in your field

- rapid publication on acceptance

- support for research data, including large and complex data types

- gold Open Access which fosters wider collaboration and increased citations

- maximum visibility for your research: over $100 \mathrm{M}$ website views per year

At BMC, research is always in progress.

Learn more biomedcentral.com/submissions 